\title{
SPEED-DEPENDENT EFFECTS ON THE 748.8 nm Ne SELF-BROADENED LINE
}

\author{
R. Ciuryzo, A. Bielski, S. Brym*, J. Domyseawska, D. Lisak, J. Szudy \\ AND R.S. TRAWIŃSKI \\ Institute of Physics, Nicholas Copernicus University \\ Grudziądzka. 5/7, 87-100 Toruń, Poland
}

(Received April 26, 1999; in final form July 13, 1999)

\begin{abstract}
Profiles of the self-broadened $748.8 \mathrm{~nm}$ Ne line emitted from a glow discharge in neon in the pressure range between 0.8 and $100 \mathrm{Tr}$ were measured using an improved experimental setup with pressure-scanned Fabry-Perot interferometer. First, line profiles were analysed using an ordinary Voigt procedure which yielded the Doppler widths decreasing with the increase in neon pressure. Using a speed-dependent Voigt profile it was shown that for the $748.8 \mathrm{~nm}$ line the correlation between the Doppler and collisional broadening plays an important role. However, the values of the pressure broadening and shift coefficients determined by the speed-dependent Voigt analysis were found to be only slightly different from those obtained by the ordinary Voigt analysis. A systematic error in the determination of perturber concentration in our previous investigation was corrected.
\end{abstract}

PACS numbers: 32.70.-n, 33.70.-w, 34.20.-b

\section{Introduction}

The role of speed-dependent effects on spectral line shapes continues to be the subject of numerous investigations. In traditional analysis, one assumes that the pressure and Doppler broadening are uncorrelated and the change of velocity during collision can be omitted. In the impact limit, the resultant line shape is then represented by a Voigt profile (VP), i.e. a convolution of a Gaussian profile arising from the thermal motion of emitters with Maxwellian distribution of velocities and a Lorentzian profile describing the collisional broadening. If, however, the speed-changing collision and the correlation between collisions and thermal motion are taken into account, then as was shown [1-9] the resultant profile may deviate significantly from the Voigt profile and give rise to an asymmetric line shape. The

*Department of Physics, Pedagogical University, Żołnierska 14, 10-561 Olsztyn, Poland. 
nature of this asymmetry is different from the asymmetry caused by collision time duration $[10,11]$ and line-mixing $[12,13]$.

Most experiments on speed-dependent effects performed in recent years dealt with profiles of molecular lines of $\mathrm{H}_{2}, \mathrm{CH}_{3} \mathrm{~F}, \mathrm{CO}, \mathrm{C}_{2} \mathrm{H}_{2}$, and $\mathrm{HF}$ molecules perturbed by various gases [14-22]. For atomic lines the only experiments in which speed-dependent effects were studied are those performed by McCartan and Lwin [8] for Li-Xe, Lewis and his group [9] for Ca-rare gas atoms, and Brym et al. [23] for Cd-Xe. They confirmed earlier theoretical predictions [5-7] that the correlation between the pressure and Doppler broadening becomes increasingly apparent with increasing values of $\alpha=m_{\mathrm{P}} / m_{\mathrm{E}}$, i.e. the ratio of the mass of perturbing atom to that of the emitting atom. It was also shown that for a system with high mass ratio $(\alpha \gg 1)$ the neglect of correlation effects may lead to significant errors in temperatures inferred from the Doppler widths. On the other hand, however, for small values of the mass ratio $(\alpha \ll 1)$ correlation effects can be always neglected. For systems with $\alpha \approx 1$ the role of the statistical dependence between the collisional and the Doppler broadening has not been, so far, sufficiently explained, although the measurements of both the Lewis group for $\mathrm{Ca}-\mathrm{Ar}$ [9] $(\alpha=1.0)$ and Brym et al. [23] for $\mathrm{Cd}-\mathrm{Xe}(\alpha=1.15)$ seem to indicate that also in these cases the correlation effects should be taken into account in order to determine the reliable line shape parameters.

In order to get to know more about the role of the Doppler-collision correlation in the formation of pressure-broadened spectral line shapes we undertook an experimental study in which a very careful analysis of the self-broadened $748.8 \mathrm{~nm}$ line of neon was performed. In this case the perturber is an atom identical to the emitting ( $\mathrm{Ne}$ ) atom so that $\alpha=1$.

The collisional effects on the $748.8 \mathrm{~nm} \quad\left(2 p^{5}\left({ }^{2} P_{3 / 2}^{0}\right) 3 p[1 / 2]_{1}-\right.$ $\left.2 p^{5}\left({ }^{2} P_{3 / 2}^{0}\right) 3 d[3 / 2]_{2}^{0}\right)$ neon line caused by ground-state Ne-atoms, i.e. for $\alpha=1$ were the subject of an earlier study from this laboratory [24] in which the profiles of this line were measured in the pressure range between 0.4 and $16.5 \mathrm{Tr}$. The coefficients of pressure broadening and shift were there determined from the clependencies of the half-width and shift of the Lorentzian component of the total profile of the $748.8 \mathrm{~mm} \mathrm{Ne}$ line on the gas pressure and it was assumed that the Lorentzian and Doppler profiles are completely uncorrelated. Contrary to that, in the present paper this assumption is not made. Our goal in the present study was to verify theoretical prediction $[5,6,25]$ that speed-dependent (correlation) effects may be also important for cases when the perturbing and emitting atoms have comparable masses, i.e. for $\alpha \approx 1$. To obtain reliable line shape parameters we extended markedly the gas pressure range ( up to $100 \mathrm{Tr}$ ) with respect to that (16.5 Tr) in the previous study [24]. Moreover, in the present experiment we were able to improve both the experimental accuracy of our setup as well as the procedures used to analyse the line profiles. In consequence, during the course of the present study we found that the values of the gas temperature and perturber density determined in the previous paper [24] are subject to a systematic error. We used several procedures and tests which permitted us to determine corrected value of both the gas temperature and gas density. 
All measurements were performed in the low density region, where two-body interactions predominate so that the impact theory is applicable. In the first approximation, the collisional component of the total line shape may be described by the Lorentzian profile characterised by the Lorentzian half-width $\gamma_{\mathrm{L}}$ and the pressure shift. $\Delta$. Both these parameters are linearly dependent on the number density $N$ of perturbing gas

$$
\begin{aligned}
& \gamma_{\mathrm{L}}=\gamma_{\mathrm{L}}^{(0)}+\beta N, \\
& \Delta=\Delta^{(0)}+\delta N .
\end{aligned}
$$

Here $\beta$ and $\delta$ denote the pressure broadening and shift coefficients, respectively, and $\gamma_{\mathrm{L}}^{(0)}$ and $\Delta^{(0)}$ are the residual Lorentzian width and shift resulting in the limit $N \rightarrow 0$.

\section{Experimental}

\subsection{Apparatus}

The experimental details have been described in the earlier papers from this laboratory $[24,26]$. The neon lines were formed in emission in a glow clischarge source which was identical to that described elsewhere [27]. Line profiles were analysed using a grating spectrograph with a pressure-scanned Fabry-Perot interferometer of the type described previously [28] with dielectric coating and a $1.513 \mathrm{~cm}$ spacer: (free spectral range $0.3305 \mathrm{~cm}^{-1}$ ). Measurements of the pressure shift of the line peak were made relative to the peak of the same line formed in a reference source which consisted of an electrodeless if neon discharge tube, and maintained under constant conditions. The pressure of neon in the reference source was kept constant at $0.8 \mathrm{Tr}$. The intensity distribution in a broadened line emitted from a glow discharge in pure neon was monitored using a photomultiplier in a photon counting mode.

Light was collected from the posilive column of the discharge, from the capillary of the diameter $2 \mathrm{~mm}$ and the length $25 \mathrm{~mm}$. The current stabilised glow discharge in pure neon could operate over the gas pressure range from 0.8 to $100 \mathrm{Tr}$. In all measurements reported in this paper which were performed in that pressure range the discharge current was always constant at. $1.5 \mathrm{~mA}$. For the discharge capillary of the diameter $2 \mathrm{~mm}$ this gives a current density of $50 \mathrm{~mA} \mathrm{~cm}^{-2}$. In our earlier paper [2.9] the dependence of the width and shift parameters of the $486.3 \mathrm{~nm}$ $\mathrm{Ne}$ line (which belongs to transitions between configuration $2 p^{5} 3 p-2 p^{5} 6 s$ ) on the glow discharge currents was studied. It was found that for current below $1.8 \mathrm{~mA}$ the width and shift were independent of the current. This means that for such currents the Stark broadening of the $486.3 \mathrm{~mm}$ can be neglected. Since the effective quantum number $n^{*}=2.99$ of the upper state $\left(2 p^{5} 3 d\right.$ l) of the $748.8 \mathrm{~mm}$ Ne line is much lower than that $\left(n^{*}=5.99\right)$ of the $486.3 \mathrm{~nm}$ line, we can conclude that also under our experimental conditions for the $748.8 \mathrm{~nm}$ Ne line for current below $1.8 \mathrm{~mA}$ the Stark broadening does not play any role. This means that under such conditions the collisional effects on the $748.8 \mathrm{~mm}$ line are mainly due to interactions of the neutral excited Ne-atoms with ground-state Ne-atoms. 


\subsection{Determination of the gas temperature}

The knowledge of the temperature of the gas in the positive column of the glow discharge is necessary to determine the atom density. The temperature of the external wall of the discharge capillary was determined with a copper-constantan thermocouple attached to that wall. For the $1.5 \mathrm{~mA}$ discharge current the temperature was found to be about $305 \mathrm{~K}$ which was nearly $10 \mathrm{~K}$ higher than the room temperature in our laboratory. The effective temperature of the emitting gas in the discharge is somewhat higher than the temperature of the external wall of the discharge capillary. Therefore the effective temperature of the emitting gas in the discharge was also determined on the basis of the measured values of the Doppler width of three selected spectral lines of argon (750.4, 703.0,687.1 mm) emitited from the $1.5 \mathrm{~mA}$ glow discharge in the Ar-He mixture [30]. Careful tiests performed at very low pressure indicated that for these lines correlation effects and excitation processes had no influence on the resultant line shape [30, 31]. The observed profiles of these lines were subject to a Voigt analysis. A crucial problem in this analysis deals with the convolution of the Voigt profile with the instrumental profile of the Fabry-Perot interferometer (FPI).

In our previous paper [24] the instrumental profile of FPI was assumed in the form of a well-known Airy profile which, in principle, can be applied to the case of an ideal FPI [32]. The analysis of the observed profile was then made in terms of convolution of the Airy function and the Voigt profile. In such a treatment the Gaussian component of the profile was treated as due entirely to the Doppler effect and was used to derive the temperature of the gas and thus its density. In the course of the present study we found that the Doppler temperatures determined in such a way seem to be too high. First of all they appear to be much higher than temperature monitored by a thermocouple attached to the external wall of the discharge tube. A thorough analysis of our experimental arrangement showed that this fact is caused by imperfections of the interferometer surfaces and maladjustment, i.e. by the fact that our FPI is not an ideal FPI. In their treatment of real FPI Jiglinskii and Kuchinskii [32] have theoretically shown that imperfections of the interferometer mirrors and misalignment of these mirrors give rise to the broadening of essentially Gaussian type and the Lorentzian correction is much smaller and can be neglected. Tests in our: laboratory confirmed their prediction. In our research, the interferometer mirrors (which were flat to better than $\lambda / 40$ ) were maintained parallel to within $\lambda / 60$.

Following Ballik [33] and Jiglinskii and Kuchinskii [32], when the Lorentzian correction to instrumental function can be neglected, the response function $O_{\mathrm{V}}(\omega)$ of a scanning FPI to a convolution of the Voigt profile and the instrumental (Airy-type) profile of a real FPI can be written in the form

$$
O_{\mathrm{V}}(\omega)=\frac{2}{\Omega}\left\{\frac{1}{2}+\sum_{n=1}^{\infty} R^{n} \mathrm{e}^{-n L} \mathrm{e}^{-n^{2}\left(G^{2}+D^{2}\right) / 4} \cos \left[\frac{2 n \pi}{\Omega}\left(\omega-\omega_{0}-\Delta\right)\right]\right\},
$$

where $\Omega$ is the free spectral range of FPI, $R$ is the reflection coefficient of the FPI mirrors, $\omega_{0}$ is the unperturbed frequency, and $\Delta$ is the pressure shift of the maximum of the line. In Eq. (3) $L=\pi \gamma_{\mathrm{L}} / \Omega$ is the reduced Lorentzian width, 
$G=\pi \gamma_{G} /(\Omega \sqrt{\ln 2})$ is the reduced width of the Gaussian component of the instrumental function of the real FPI, and $D=\pi \gamma_{\mathrm{D}} /(\Omega \sqrt{\ln 2})$ is the reduced Doppler width. The Doppler width $\gamma_{\mathrm{D}}$ which depends on the gas temperature $T$, the mass $m_{\mathrm{E}}$ of emititing atom, and the unperturbed frequency $\omega_{0}$ is given by $\gamma_{\mathrm{D}}=$ $k v_{m_{\mathrm{E}}} 2 \sqrt{\ln 2}$ where $k=\omega_{0} / c, v_{m_{\mathrm{E}}}=\sqrt{2 k_{\mathrm{B}} T / m_{\mathrm{E}}}$ is the most probable speed of emitter and $k_{\mathrm{B}}$ is Boltzmann's constant.

Using a procedure described in Ref. [32] we found that in case of our FPI the instrumental contribution $\gamma_{\mathrm{G}}$ to Gaussian width accounts for about $0.010 \mathrm{~cm}^{-1}$. On the basis of such an analysis we determined the Doppler widths of three selected argon lines. These Doppler widths were then used to obtain the effective temperature of the emitting gas in the discharge. For the $1.5 \mathrm{~mA}$ current the gas temperature was found to be $(320 \pm 20) \mathrm{K}$. This value of temperature was then used to determine the number density $N$ of the gas. Using these values of $N$ the pressure broadening $(\beta)$ and shift $(\delta)$ coefficients were determined on the basis of Eqs. (1) and (2).

It should be emphasised that in an analysis reported in Ref. [24] the instrumental contribution $\gamma_{G}$ to the total Gaussian width has not been taken into account assuming that the Gaussian width is identical to the Doppler width. This means that the gas temperature $T^{*}$ reported in Ref. [24] is too high. During the course of the present study we verified that under conditions of experiments described in Ref. [24] the real gas temperature in the discharge region at $1.5 \mathrm{~mA}$ is close to $(320 \pm 20) \mathrm{K}$. We should note, however, that both the number densities and the pressure broadening $(\beta)$ and shift $(\delta)$ coefficients reported in [24] can be easily corrected by multiplying those values by a factor of $(320 \mathrm{~K}) / T^{*}$.

\section{Line shape analysis}

The response of the real FPI to the Voigt profile, Eq. (3), which was used by us as the basis of the determination of the gas temperature can be applied to cases when the speed-dependent effects such as Doppler-collision correlation can be ignored, i.e. for a system where the emitter is much heavier than the perturber $(\alpha \ll 1)$. In the present paper on the self-broadening of the $748.8 \mathrm{~nm} \mathrm{Ne}$ line our goal is to check the possibility of the appearance of correlation effects for $\alpha=1$. As the starting point of our analysis we adopt here the speed-dependent Voigt profile (SDVP) in the form derived by Berman [5]

$$
I_{\mathrm{SDV}}(\omega)=\frac{1}{2 \pi} \int \mathrm{d}^{3} v_{\mathrm{E}} f_{m_{\mathrm{E}}}\left(v_{\mathrm{E}}\right) \frac{\gamma_{\mathrm{L}}\left(v_{\mathrm{E}}\right)}{\left[\omega-\omega_{0}-k v_{\mathrm{E}}-\Delta\left(v_{\mathrm{E}}\right)\right]^{2}+\left[\gamma_{\mathrm{L}}\left(v_{\mathrm{E}}\right) / 2\right]^{2}},
$$

where

$$
f_{m_{\mathrm{E}}}\left(v_{\mathrm{E}}\right)=\left(\frac{m_{\mathrm{E}}}{2 \pi k_{\mathrm{B}} T}\right)^{\frac{3}{2}} \exp \left(-\frac{m_{\mathrm{E}} v_{\mathrm{E}}{ }^{2}}{2 k_{\mathrm{B}} T}\right)
$$

is the Maxwellian distribution of velocities $v_{\mathrm{E}}$ of the emitting atoms with mass $m_{\mathrm{E}}$. In Eq. (4) $\gamma_{\mathrm{L}}\left(v_{\mathrm{E}}\right)$ and $\Delta\left(v_{\mathrm{E}}\right)$ are the speed-dependent Lorentzian width and pressure shift of the impact theory, respectively. Assuming the validity of the classical-path approximation Ward et al. [6] have shown that in the adiabatic case of isolated spectral lines $\gamma_{\mathrm{L}}\left(v_{\mathrm{E}}\right)$ and $\Delta\left(v_{\mathrm{E}}\right)$ can be written as 


$$
\begin{aligned}
& \frac{\gamma_{\mathrm{L}}\left(v_{\mathrm{E}}\right)}{2}+\mathrm{i} \Delta\left(v_{\mathrm{E}}\right)=2 \pi N \int \mathrm{d}^{3} v_{\mathrm{EP}} f_{m_{\mathrm{P}}}\left(v_{\mathrm{E}}+v_{\mathrm{EP}}\right) v_{\mathrm{EP}} \\
& \quad \times \int_{0}^{+\infty} \mathrm{d} \rho \rho\left\{1-\exp \left[-\mathrm{i} \eta\left(\rho, v_{\mathrm{EP}}\right)\right]\right\}
\end{aligned}
$$

where

$$
\eta\left(\rho, v_{\mathrm{EP}}\right)=\frac{1}{\hbar} \int_{-\infty}^{+\infty} \Delta V(r(t)) \mathrm{d} t
$$

is the phase-shift function of the classical impact theory $[34,35]$ and $\Delta V(r(t))$ is the difference of interaction potentials between perturbing and emitting atoms in its upper and lower state. Here $r(t)=\sqrt{\rho^{2}+v_{\mathrm{EP}}^{2} t^{2}}$ is the distance between emitting and perturbing atoms at time $t . v_{\mathrm{EP}}$ is the mean relative velocity and $f_{m_{\mathrm{P}}}\left(v_{\mathrm{E}}+v_{\mathrm{EP}}\right)$ is the Maxwellian distribution of the perturber velocities $v_{\mathrm{P}}=$ $v_{\mathrm{E}}+v_{\mathrm{EP}}$. It should be noted that the average of $\gamma_{\mathrm{L}}\left(v_{\mathrm{E}}\right)$ and $\Delta\left(v_{\mathrm{E}}\right)$ over all emitter velocities yields the width $\gamma_{\mathrm{L}}$ and the shift $\Delta$ of the conventional line broadening treatment

$$
\begin{aligned}
& \gamma_{\mathrm{L}}=\int \mathrm{d}^{3} v_{\mathrm{E}} f_{m_{\mathrm{E}}}\left(v_{\mathrm{E}}\right) \gamma_{\mathrm{L}}\left(v_{\mathrm{E}}\right), \\
& \Delta=\int \mathrm{d}^{3} v_{\mathrm{E}} f_{m_{\mathrm{E}}}\left(v_{\mathrm{E}}\right) \Delta\left(v_{\mathrm{E}}\right) .
\end{aligned}
$$

Following Ward et al. [6] we introduce dimensionless width $B_{\mathrm{W}}(x ; \alpha)$ and shift $B_{\mathrm{S}}(x ; \alpha)$ functions which are defined by

$$
B_{\mathrm{W}}(x ; \alpha)=\frac{\gamma_{\mathrm{L}}\left(x v_{m_{\mathrm{E}}}\right)}{\gamma_{\mathrm{L}}}
$$

and

$$
B_{\mathrm{S}}(x ; \alpha)=\frac{\Delta\left(x v_{m_{\mathrm{E}}}\right)}{\Delta},
$$

where $x=v_{\mathrm{E}} / v_{m_{\mathrm{E}}}$ is the reduced speed of emitter. Here $\gamma_{\mathrm{L}}\left(x v_{m_{\mathrm{E}}}\right) \equiv \gamma_{\mathrm{L}}\left(v_{\mathrm{E}}\right)$ and $\Delta\left(x v_{m_{\mathrm{E}}}\right) \equiv \Delta\left(v_{\mathrm{E}}\right)$ are defined by Eq. (6).

As we verified during the course of this study the imperfections of the interferometer mirrors and misadjustment give rise to the appearance of the Gaussian component of the FPI instrumental profile. Using the transformations similar to those applied to derive $\mathrm{Eq}$. (3) one can show that the response $\mathrm{O}_{\mathrm{SDV}}(\omega)$ of the real FPI to the speed-dependent Voigt profile, Eq. (4), can be written in the form $[30,31]$

$$
O_{\mathrm{SDV}}(\omega)=\frac{2}{\Omega}\left[\frac{1}{2} F_{0}(\omega)+\sum_{n=1}^{\infty} R^{n} \exp \left(-\frac{G^{2} n^{2}}{4}\right) F_{n}(\omega)\right]
$$

where

$$
\begin{aligned}
F_{n}(\omega) & =\frac{4}{\sqrt{\pi}} \int_{0}^{+\infty} \mathrm{d} x x^{2} \mathrm{e}^{-x^{2}} \operatorname{Sinc}(x D n) \exp \left[-L B_{\mathrm{W}}(x, \alpha) n\right] \\
\times & \cos \left\{\frac{2 n \pi}{\Omega}\left[\omega-\omega_{0}-\Delta B_{\mathrm{S}}(x, \alpha)\right]\right\} .
\end{aligned}
$$


Here $\operatorname{Sinc}(y)=\sin (y) / y$. In the limit when correlation effects can be neglected we have $B_{\mathrm{W}}(x ; \alpha)=B_{\mathrm{S}}(x ; \alpha)=1$ and then the response $O_{\mathrm{SDV}}(\omega)$ of the FPI to the speed-dependent Voigt profile becomes identical to the response $O_{\mathrm{V}}(\omega)$ to the ordinary Voigt profile, Eq. (3). On the other hand, in the case of an ideal FPI when $G=0$, Eq. (3) can be transformed to the expression given in Ref. [25].

In the present study the measured line profiles were fitted first to the response function $O_{\mathrm{V}}(\omega)$ of the real FPI to the ordinary Voigt profile, Eq. (3), and then to the speed-clependent Voigt profile $O_{\operatorname{SDV}}(\omega)$ given by Eq. (13). The fitting parameters were Lorentzian widlh $\gamma_{\mathrm{L}}$, the Doppler width $\gamma_{\mathrm{D}}$, and the pressure shift $\Delta$. The values of these parameters were determined by a least-squares fit of the measured interferograms at various clensities to either Eq. (3) or Eq. (13) using an algorithm given by Marquardt [36].

\section{Results}

\subsection{The Voigt analysis}

All results described below were obtained from measurements of the self-broadened profiles of the $748.8 \mathrm{~mm}$ Ne line enitted from a glow discharge in pure neon in which the current was maintained constant at $1.5 \mathrm{~mA}$ and the neon pressure was varied in the region between 0.79 and $100 \mathrm{Tr}$.

The profiles were first analysed in terms of the ordinary Voigt profile assuming that the correlation between the Doppler and collision broadening can be neglected. The values of the Doppler width $\gamma_{\mathrm{D}}$ determined from the best fit of the interferograms of the $748.8 \mathrm{~nm}$ line to the Voigt response function $O_{V}(\omega)$, Eq. (3), are shown in Fig. 1a, where they are plotted against the pressure of neon in the source. The Doppler width $\gamma_{\mathrm{D}}^{(0)}$ corresponding to the gas temperature $320 \mathrm{~K}$ in the discharge capillary is about $38 \times 10^{-3} \mathrm{~cm}^{-1}$. As is seen, the Doppler widths obtained from the Voigt analysis were found to be below $\gamma_{D}^{(0)}$ as the pressure increased. This result cannot be attributed to thermalization processes [37, 38], because they can lead only to stabilisation of the Doppler width on the level close to $38 \times 10^{-3} \mathrm{~cm}^{-1}$ contrary to our observation.

The observed decrease $(5.0 \pm 1.5) \times 10^{-3} \mathrm{~cm}^{-1}$ in the Doppler width also cannot be explained by the Dicke narrowing [1]. Using a diffusion model put forward first by Galatiry [2] and developed recently [39] we estimated, in the way described by Bielski et al. [31], that for the $748.8 \mathrm{~nm}$ self-broadened $\mathrm{Ne}$ line the value of the decrease in the Doppler width caused by the Dicke narrowing should be smaller than $1.5 \times 10^{-3} \mathrm{~cm}^{-1}$ under our experimental conditions. To estimate the value of the effective velocity-changing collision rate we assumed that $\mathrm{Ne}^{*}$ and Ne-atoms collide as hard spheres with effective diameter about $3 \AA$. This diameter value seems to be realistic comparing potential curves for $\mathrm{Ne}^{*}-\mathrm{Ne}$ interaction in other excited states [40]. It should be noted that the Lennard-Jones potential $V(r)=C_{12} r^{-12}-C_{6} r^{-5}$ with force constant $C_{12}$ obtained on the basis of an expression given by Hindmarsh et al. [41], used to describe interaction in higher excited states, appears to be much more repulsive than more realistic potentials [40]. The value of the effective velocity-changing collision rate obtained 


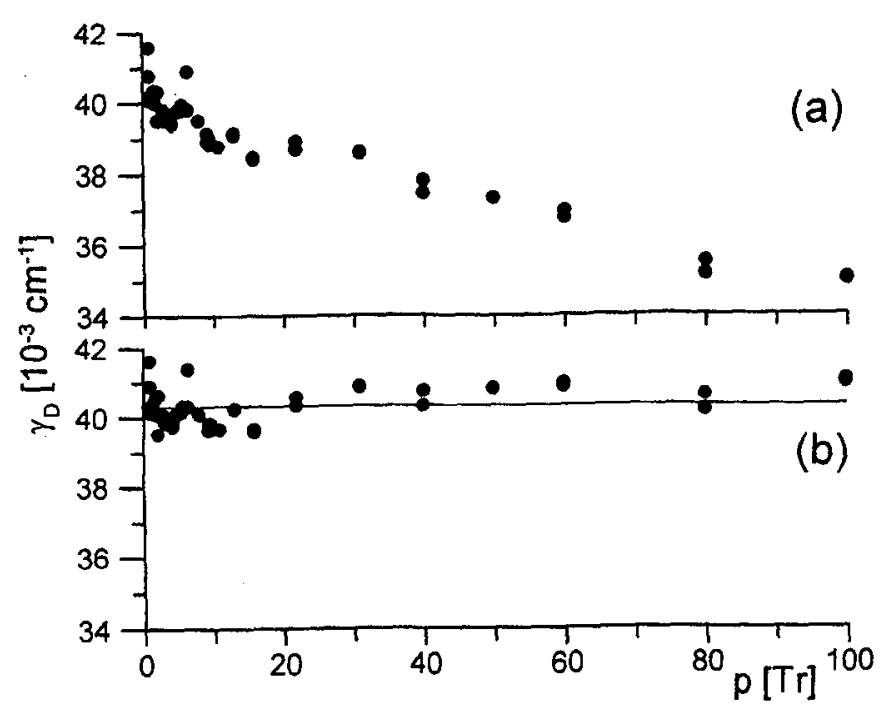

Fig. 1. Plots of the Doppler width $\gamma_{\mathrm{D}}$ (FWHM) of the self-broadened $748.8 \mathrm{~mm}$ Ne line against the neon pressure $p$ determined for different fitting profiles: (a) $O_{\vee}(\tilde{\nu})$ profile, (b) $O_{S D V}(\widetilde{\nu})$ profile calculated for the van der Waals potential. The mean value of $\gamma_{D}$ is marked by the solid line (-).

using such a Lemnard-Jones potential can be much higher than the value obtained using more realistic potentials. Nevertheless, such a. Lennard-Jones potential may give rise to quite good values of pressure broadening and shift coefficients. Taking into account the estimated value (about $1.5 \times 10^{-3} \mathrm{~cm}^{-1}$ ) of the Dicke narrowing and the fact that the correlation between velocity-changing and clephasing collisions $[4,18,42,22]$ may lead to complete reduction of the Dicke effect [18], we can conclude that the observed decrease in the Doppler width should not be caused by the Dicke narrowing effect.

Such a reduction of the Doppler width with increasing pressure was first experimentally observed for the xenon-perturbed Li-resonance line by McCartan and Lwin [8] who explained this result as an effect caused by the correlations between the thermal motion and pressure broadening. Their case, however, is the most extreme one when the masses of the perturber and the emitter are as different as possible $(\alpha=22)$, but as noted by Lewis and his co-workers [9] that effect should be observed in a great range of cases corresponding to much smaller values of the mass ratio $\alpha$.

The above discussion shows that thermalisation processes as well as velocity-changing collisions cannot explain the observed decrease in the Doppler width. Therefore, according to Berman [5] and Ward et al. [6] the pressure-dependent narrowing of the Doppler component of the Voigt profile of the $748.8 \mathrm{~nm}$ self-broadened neon line which is clearly seen in Fig. 1a may thus be regarded as an experimental evidence that speed-dependent effects leading to the correlation between the Doppler and collisional broadening can also be present for systems with the mass ratio $\alpha$ close to 1 . 


\subsection{The speed-dependent Voigt analysis}

The apparent reduction in the Doppler width with increasing pressure presented in Fig. 1a means that the ordinary Voigt profile fails due to the neglect of the correlation effects. In order to take into account these effects we use here the speed-dependent Voigt profile, Eq. (4). The measured interferograms of the self-broadened $748.8 \mathrm{~nm}$ Ne line were then fitted to the function $O_{\mathrm{SDV}}(\omega)$, Eq. (12), which represents the response of the real FPI to the speed-dependent Voigt profile. To compute the profiles from Eq. (12) the reduced broadening and shift functions $B_{W}(x ; \alpha)$ and $B_{\mathrm{S}}(x ; \alpha)$ must first be calculated. This can be done provided the interaction potentials in the upper and lower state of the emitter are known.

In the present paper we used the adiabatic approach due to Lindholm and Foley $[43,34]$ assuming the interaction potentials for the upper and lower state in the form of a van der Waals potential $V(r)=-C_{6} r^{-6}$. The values of force constant $C_{6}$ for states under investigation were obtained using the Unsold approximation [44]. They are equal to 291 au and 83 au for the upper and lower state, respectively.

Ward et al. [6] have shown that for the van der Waals potential the following relation is fulfilled:

$$
B_{\mathrm{W}}(x ; \alpha)=B_{\mathrm{S}}(x ; \alpha) \equiv(1+\alpha)^{-0.3} M\left(-0.3,1.5,-\alpha x^{2}\right),
$$

where $M(a, b, z)$ is the confluent hypergeometric function. As is seen from Eq. (14), for the van der Waals potential the reduced broadening $\left(B_{\mathrm{W}}(x, \alpha)\right)$ and shift $\left(B_{\mathrm{S}}(x, \alpha)\right)$ functions are identical and independent of force constant. For other potentials these functions may differ markedly as was shown by Pine [22] in the case of $H F$ lines perturbed by $\operatorname{Ar}(\alpha=2)$.

As the next step in our analysis we used the $B_{\mathrm{W}}(x, \alpha)$ and $B_{\mathrm{S}}(x, \alpha)$ functions to fit the experimental profiles to the speed-dependent Voigt response function, Eq. (12). The values of the Doppler width $\gamma_{D}$ determined from such a fit plotted versus the neon pressure are shown in Fig. 1b for the case of the van der Waals potential.

As is seen from Fig. 1b, the line shape analysis based on speed-dependent Voigt profile yields the Doppler widths $\gamma_{D}$ which are practically independent of the neon pressure. The mean value $\bar{\gamma}_{D}$ determined from the plot shown in Fig. 1b was found to be $(40.3 \pm 1.5) \times 10^{-3} \mathrm{~cm}^{-1}$, which is close to the value $(38.3 \pm 1.3) \times 10^{-3} \mathrm{~cm}^{-1}$ corresponding to the Doppler temperature $(320 \pm 20) \mathrm{K}$ of the gas in the clischarge capillary. We should note, however, that $\bar{\gamma}_{\mathrm{D}}$ obtained from our analysis is above $\gamma_{\mathrm{D}}^{(0)}$.

Figure 2a shows an example of the measured profile of the self-broadened $748.8 \mathrm{~nm} \mathrm{Ne}$ line recorded at $80 \mathrm{Tr}$. The weighted differences $D_{\sigma}(\omega)=$ $\left[I_{\exp }(\omega)-I_{\text {fit }}(\omega)\right] / \sqrt{I_{\text {fit }}(\omega)}$ between the experimental $I_{\exp }(\omega)$ and the best fit $I_{\text {fit }}(\omega)$ value obtained from the ordinary Voigt response, Eq. (3), are plotted in Fig. 2b. Similar plots obtained from the speed-dependent Voigt profile, Eq. (12), are presented in Fig. 2c. It should be noted that in the case of the van der Waals potential the use of the speed-dependent Voigt profile as the basis of the fitting procedure does not lead to the improvement of the best fit (Fig. 2c) in compar- 

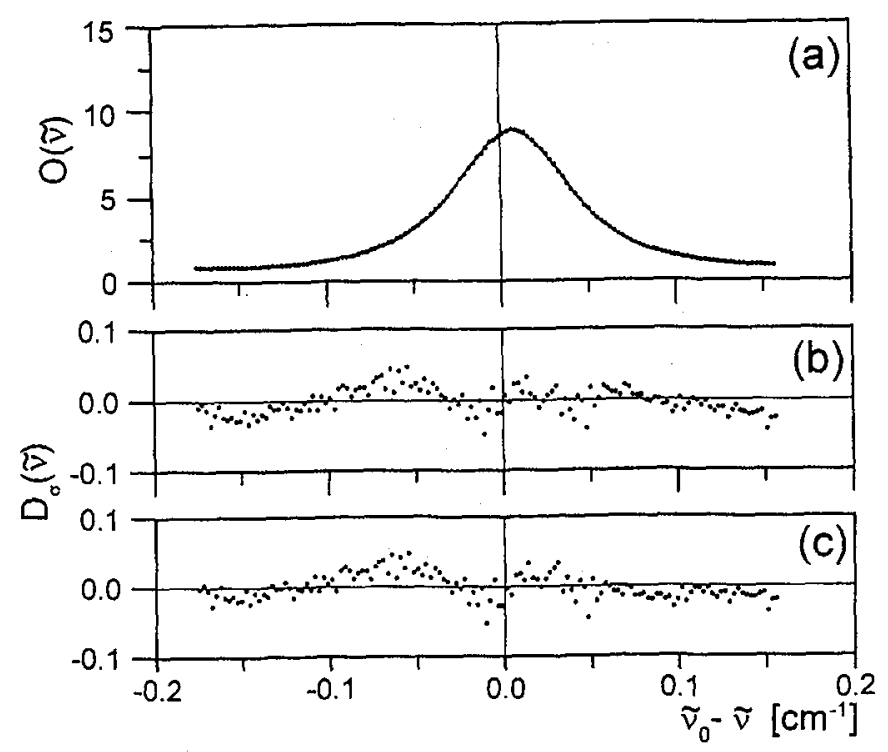

Fig. 2. (a) Shape of the self-broadened $748.8 \mathrm{~nm}$ Ne line at neon pressure $80 \mathrm{Tr}$, (•) experimental points, solid line (-) the best fit. $O_{V}(\widetilde{\nu})$ profile (in relative units). (b) Weighted differences $D_{\sigma}(\tilde{\nu})$ between the observed profile and the fitted $O_{\vee}(\tilde{\nu})$ profile (in relative units). (c) Weighted differences $D_{\sigma}(\tilde{\nu})$ between the observed profile and the fitted $O_{\text {SDV }}(\widetilde{\nu})$ profile (in relative units) calculated for the van der Waals potential.

ison to that illustrated in Fig. 2b. Most probably such a result is caused by the fact that the van der Waals potential does not describe a repulsive interaction for small interatomic separations and is insufficient to give a correct description of speed-clependent collisional effects [22].

The values of the Lorentzian width $\gamma_{\mathrm{L}}$ and shift $\Delta$ determined from the fits of our experimental profile to the Voigt response, Eq. (3), are shown in Fig. 3, where they are plotted against the number density $N$ of neon. It is interesting to note that the values $\gamma_{\mathrm{L}}$ and $\Delta$ obtained from speed-dependent analysis are so close to those derived from the Voigt analysis that they cannot be distinguished from them in Fig. 3. Therefore in Fig. 3 we put only the values of $\gamma_{L}$ and $\Delta$ obtained from the Voigt analysis. Figure 3 shows that $\gamma_{\mathrm{L}}^{(0)}$ and $\Delta^{(0)}$ are not equal to zero. The nonzero value of $\gamma_{\mathrm{L}}^{(0)}$ is caused by a. Lorentzian component of the instrumental function which is not consiclered in our analysis as well as by the effects due to excitation processes [26]. The nonzero value of $\Delta^{(0)}$ is caused by the fact that our shift measurements were performed in such a way that we measured the position of the maximum of the spectral line emitted by the glow discharge source with respect to that of the same line emitted by reference source.

The pressure broadening $(\beta)$ and shift $(\delta)$ coefficients defined by Eqs. (1)-(2) determined from the slopes of straight lines plotted in Fig. 3 are listed in Table, where the values determined from the fits to the Voigt profile, Eq. (3), are marked by "VP". In Table the values marked by "SDVP" are the coefficients determined from the fits to the speed-dependent Voigt profile, Eq. (12), using the van der Waals 


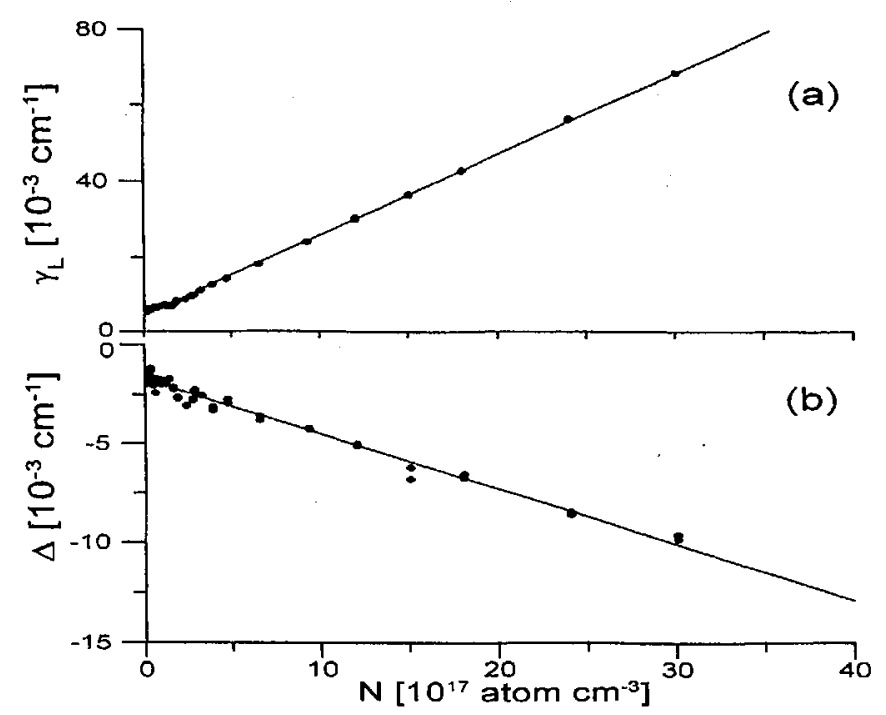

Fig. 3. Plots of (a) the Lorentzian width (FWHM) $\gamma_{\mathrm{L}}$ and (b) shift $\Delta$ for the $748.8 \mathrm{~nm}$ Ne line against the perturbing gas density $N$ ( $\bullet$ experimental points) determined using the $O_{V}(\widetilde{\nu})$ profile. Straight lines (- - ) represent a least-squares fit of experimental points to linear dependence of $\gamma_{\mathrm{L}}$ and $\Delta$ on $N$.

potential. An important result of this paper is that the values of the broadening and shift coefficients determined by the speed-dependent Voigt profile analysis are only slightly different, about $2-3 \%$, from those obtained by the ordinary Voigt analysis.

TABLE

Comparison of experimental values of the $\beta$ and $\delta$ coefficients (in units $10^{-20} \mathrm{~cm}^{-1} /$ atom $\mathrm{cm}^{-3}$ ) of the $748.8 \mathrm{~nm}$ neon line perturbed by neon determined on the basis of the ordinary Voigt (VP) and speed-dependent Voigt (SDVP) procedures with experimental results of Trawinski et al. [24] and with theoretical valnes for the van der Waals potential (vdW). For experimental data the values of standard deviations are given.

\begin{tabular}{c|c|c|c}
\hline \hline Experimental & $\beta$ & $\delta$ & $\delta / \beta$ \\
\hline VP & $2.124 \pm 0.011$ & $-0.280 \pm 0.006$ & $-0.132 \pm 0.003$ \\
SDVP & $2.081 \pm 0.013$ & $-0.288 \pm 0.006$ & $-0.139 \pm 0.003$ \\
Trawiński et al. [24] & $2.26 \pm 0.12$ & $-0.29 \pm 0.04$ & $-0.13 \pm 0.01$ \\
& $(1.82 \pm 0.10)$ & $(-0.23 \pm 0.04)$ & \\
\hline Theoretical & $\beta$ & $\delta$ & $\delta / \beta$ \\
\hline vdW & 1.913 & -0.695 & -0.363
\end{tabular}


Table also contains the experimental values of $\beta$ and $\delta$ coefficients determined by Trawiński et al. [24] in a previous paper from this laboratory. These values were obtained on the basis of ordinary Voigt analysis. In their analysis the imperfections of the interferometer plates were not taken into account and the instrumental contribution to the Gaussian width was neglected. Assuming that the Gaussian width is entirely the Doppler width, the gas temperature for the current of $1.5 \mathrm{~mA}$ was found to be $(397 \pm 15) \mathrm{K}$. Using this value of the Doppler temperature the values of $\beta$ and $\delta$ were determined in Ref. [24]. In the present work we corrected these values by inclusion of the Gaussian part of the instrumental function. For the discharge current of $1.5 \mathrm{~mA}$ we found the corrected value of the gas temperature to be close to $320 \mathrm{~K}$ for conditions corresponding to those in Ref. [24]. The corrected values of $\beta$ and $\delta$ coefficients determined from the ordinary Voigt analysis under conditions of experiment by Trawiński et al. [24] are also listed in Table in parenthesis. Some disagreement between the corrected values of $\beta$ and $\delta$ coefficients determined by Trawiński et al. [24] and results marked by "VP" in Table is caused by the fact that the pressure range of perturber gas used in our earlier paper [24] is much narrower than in the present one.

In Table we have also listed the theoretical values of $\beta$ and $\delta$ coefficients calculated from the impact adiabatic theory due to Lindholm and Foley $[43,34]$ using the van der Waals potential. The agreement of theoretical values with measured ones is poor in the case of pressure shift. To obtain better agreement between theoretical and experimental values it is necessary to carry out semiclassical or quantum close-coupling calculations such as those recently done by Leo et al. [45] for helium and other lines of neon.

\section{Conclusion}

The theoretical predictions due to Berman [5] and Ward et al. [6] were verified for the case of the self-broadened $748.8 \mathrm{~nm} \mathrm{Ne}$ line. It was shown that even for systems with the perturber-emitter mass ratio $\alpha=1$ the effects of correlation between the broadening induced by the thermal motion and collisional broadening have a noticeable influence on the resultant line shape. In our case the Dicke narrowing is negligible and the correlation effects are found to be responsible for noticeable narrowing of the Doppler component of the resultant line shape in the case of traditional. Voigt analysis.

It should be emphasised that the quality of the fitting procedure depends on the type of the interaction potential function, $V(r)$, used to calculate the functions $B_{\mathrm{W}}(x, \alpha)$ and $B_{\mathrm{S}}(x, \alpha)$. This means that the speed-dependent narrowing of the Gaussian component of the total line shape and quality of the fit of the experimental profile to the assumed profile may serve as an additional source of information on the interatomic interactions. To describe fully speed-dependent effects on the self-broadened 748.8 neon line, semiclassical or close-coupling calculations [45] of the broadening and shift coefficients based on realistic interaction potentials for $\mathrm{Ne}^{*}-\mathrm{Ne}$ are required to evaluate more realistic functions $B_{\mathrm{W}}(x, \alpha)$ and $B_{\mathrm{S}}(x, \alpha)$. Unfortunately, such calculations have not been done as yet. 


\section{Acknowledgment}

This work was supported by a grant No. 673/P03/96/10 (2 P03B 005 10) from the Committee for Scientific Research.

\section{References}

[1] R.H. Dicke, Phys. Rev. 89, 472 (1953); J.P. Wittke, R.H. Dicke, Phys. Rev. 103, 620 (1956).

[2] L. Galatry, Phys. Rev. 122, 1218 (1961).

[3] M. Nelkin, A. Ghatak, Phys. Rev. 135, A4 (1964).

[4] S.G. Rautian, I.I. Sobelman, Usp. Fiz. Nauk 90, 209 (1966) [Sov. Phys. Uspiekhi 9, $701(1967)]$.

[5] P.R. Berman, J. Quant. Spectrosc. Radiat. Transf. 12, 1331 (1972).

[6] J. Ward, J. Cooper, E.W. Smith, J. Quant. Spectrosc. Radiat. Transf. 14, 555 (1974).

[7] E. Bielicz, E. Czuchaj, J. Fiutak, Acta Phys. Pol. A 41, 327 (1972).

[8] D.G. McCartan, N. Lwin, J. Phys. B 10, L17 (1972).

[9] E.L. Lewis, Spectral Line Shapes, Ed. J. Szudy, Vol. 5, Ossolineum, Wrocław 1988, p. 485; M. Harris, E.L. Lewis, D. McHugh, I. Shannon, J. Phys. B 17, L661 (1984); I. Shannon, M. Harris, D.R. McHugh, E.L. Lewis, J. Phys. B 19, 1409 (1986); M. Harris, E.L. Lewis, D. McHugh, I. Shannon, J. Phys. B 19, 3207 (1986).

[10] P.W. Anderson, J.D. Talman, Bell Teleph. Syst. Tech. Publ. No. 3117, University of Pittsburg, 1955 (unpublished).

[11] J. Szudy, W.E. Baylis, Phys. Rep. 266, 127 (1996).

[12] M. Baranger, Phys. Rev. 111, 494 (1958).

[13] A.S. Pine, J. Quant. Spectrosc. Radiat. Transf. 57, 145 (1997).

[14] R.L. Farrow, L.A. Rahn, G.O. Sitz, G.J. Rasasco, Phys. Rev. Lett. 63, 746 (1989).

[15] D. Robert, J.M. Thuet, J. Bonamy, S. Temkin, Phys. Rev. A 47, R771 (1993).

[16] F. Rohart, F. Mäder, H.W. Nicolaisen, J. Chem. Phys. 101, 6475 (1994); F. Rohart, A. Ellendt, F. Kaghat, H. Mäder, J. Mol. Spectrosc. 185, 222 (1997).

[17] P. Duggan, P.M. Sinclair, A.D. May, J.R. Drummond, Phys. Rev. A 51, 218 (1995).

[18] P. Duggan, P.M. Sinclair, R. Berman, A.D. May, J.R. Drummond, J. Mol. Spectrosc. 186, 90 (1997).

[19] A. Henry, D. Hurtmans, M. Margottin-Maclou, A. Valentin, J. Quant. Spectrosc. Radiat. Transf. 56, 647 (1996).

[20] P.M. Sinclair, J.Ph. Berger, X. Michaut, R. Saint-Loup, R. Chaux, H. Berger, J. Bonamy, D. Robert, Phys. Rev. A 54, 402 (1996).

[21] B. Lance, G. Blanquet, J. Walrand, J.P. Bouanich, J. Mol. Spectrosc. 185, 262 (1997).

[22] A.S. Pine, J. Quant. Spectrosc. Radiat. Transf. 62, 397 (1999).

[23] S. Brym, R. Ciuryło, R.S. Trawiński, A. Bielski, Phys. Rev. A 56, 4501 (1997).

[24] R.S. Trawiński, A. Bielski, R. Ciuryło, J. Szudy, Ann. Phys. 2, 1 (1993).

[25] R. Ciuryło, A. Bielski, S. Brym, J. Jurkowski, J. Quant. Spectrosc. Radiat. Transf. 53, 493 (1995). 
[26] R. Ciuryło, A. Bielski, J. Domysławska, J. Szndy, R.S. Trawinski, J. Phys. B 27, 4.181 (1994).

[27] A. Bielski, J. Wolnikowski, Acta Phys. Pol. A 54, 601 (1978).

[28] A. Bielski, W. Dokurno, E. Lisicki, Z. Turło, Opt. Appl. 9, 151 (1981).

[29] A. Bielski, S. Brym, J. Szudy, R.S. Trawiński, J. Wolnikowski, J. Phys. B 24, 4909 (1991).

[30] R. Ciuryło, Ph.D. thesis, Nicholas Copernicus University, Torun 1998 (in Polish).

[31] A. Bielski, S. Brym, R. Ciuryło, J. Szndy, Eur. Phys. J. D, in press.

[32] A.G. Jiglinskii, V.V. Kuchinskii, Real Fabry-Perot Interferometer, Mashinostroyenye, Leningrad 1983 (in Russian).

[33] E.A. Ballik, Appl. Opt. 5, 170 (1966).

[34] H.M. Foley, Phys. Rev. 69, 616 (1946).

[35] N. Allard, J. Kielkopf, Rev. Mod. Phys. 54, 1103 (1982).

[36] D.W. Marquardt, J. Soc. Industr, Appl. Math. 11, 431 (1963).

[37] A.R. Malvern, J.L. Nicol, D.N. Stacey, J. Phys. B 7, L518 (1974).

[38] D.N. Stacey, R.C. Thompson, J. Phys. B 16, 537 (1983).

[39] R. Ciuryło, J. Szudy, J. Quant. Spectrosc. Radiat. Transf. 57, 411 (1997).

[40] P.J. Leo, D.F.T. Mullamphy, G. Peach, V. Venturi, I.B. Whittingham, J. Phys. B 30, 535 (1997).

[41] W.R. Hindmarsh, A.D. Petford, G. Smith, Proc. R. Soc. Lond. A 247, 296 (1967).

[42] A.S. Pine, J. Chem. Phys. 101, 3444 (1994).

[43] E. Lindholm, Arkiv. Mat. Astr. Fys. A 32, No. 17 (1945).

[44] A. Unsöld, Physik der Sternatmosphären, Springer, Berlin 1983.

[45] P.J. Leo, D.F.T. Mullamphy, G. Peach, I.B. Whittingham, J. Phys. B 25, 1161 (1992); J. Phys. B 28, 4449 (1995); P.J. Leo, G. Peach, I.B. Whittingham, J. Phys. $B$ 28, 591 (1995); P.J. Leo, D.F.T. Mullamphy, G. Peach, V. Venturi, I.B. Whittingham, J. Phys. B 29, 4573 (1996); Acta Phys. Pol. A 93, 459 (1998). 